\title{
Perfil espermático de touros da raça Sindi
}

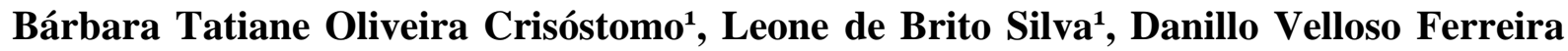 Murta $^{2 *}$, Roberta Veloso Rebello ${ }^{3}$, Silene Maria Barreto ${ }^{4}$, Lucélia Karoline Gonçalves Barbosa $^{1}$, Daniele Carolina Rodrigues Xavier ${ }^{1}$, Lucas Aquino Rodrigues ${ }^{1}$, José Alcides de Castro Machado Ribeiro ${ }^{1}$}

\author{
${ }^{1}$ Acadêmico. Graduando do Curso de Medicina Veterinária das Faculdades Integradas do Norte de Minas - FUNORTE. \\ Montes Claros, MG, Brasil. \\ ${ }^{2}$ Doutor. Docente do Curso de Medicina Veterinária das Faculdades Integradas do Norte de Minas - FUNORTE. Montes \\ Claros, MG, Brasil. \\ ${ }^{3}$ Mestranda. Universidade Estadual de Montes Claros - UNIMONTES. Montes Claros, MG, Brasil. \\ ${ }^{4}$ Mestre. Docente do Curso de Medicina Veterinária das Faculdades Integradas do Norte de Minas - FUNORTE. Montes \\ Claros, MG, Brasil. \\ *Autor para correspondência, Endereço: Rua São João - N²74. Bairro: Todos os Santos. Cidade: Montes Claros, MG. \\ CEP: 39400-126.E-mail: danillo.murta.vet@gmail.com
}

RESUMO. O exame andrológico é uma importante ferramenta na seleção de touros melhoradores do rebanho, pois possibilita a identificação de problemas de infertilidade e subfertilidade dos machos, maximizando o uso destes animais na estação de monta e melhorando consideravelmente os índices de prenhez do plantel. O propósito deste trabalho foi descrever o perfil espermático de touros da raça Sindi, de uma fazenda na cidade de São João da Ponte, no norte de Minas Gerais. Foram avaliados 11 touros separados em dois grupos $\mathrm{G} 1<36$ meses $(\mathrm{n}=6)$ e $\mathrm{G} 2>36$ meses $(\mathrm{n}=5)$ criados a pasto. Os animais foram inicialmente pesados e identificados, mensurada a circunferência escrotal, onde se obteve uma média de $33,8 \mathrm{~cm}$ para o $\mathrm{G} 1$ e de $38,2 \mathrm{~cm}$ para o $\mathrm{G} 2$ e, posteriormente, foi realizada a coleta do sêmen com o uso do eletroejaculador. O ejaculado foi submetido à análise microscópica para avaliação de turbilhonamento motilidade, e vigor obtendo-se os valores de motilidade de 78,4 e $65 \%$, vigor 3,5 e 2,6 e turbilhonamento 3 e 2,4 para G1 e G2 respectivamente. Uma amostra deste ejaculado, foi submetida a exame de morfologia espermática, utilizando-se o corante rosa bengala em preparação úmida e a técnica de esfregaço utilizando o corante hematoxilina eosina. Os resultados obtidos para o G1 foram 1,8\% de defeitos menores e 7,8\% de defeitos maiores na técnica com o corante rosa bengala e $1,2 \%$ e $12,3 \%$ de defeitos maiores e menores respectivamente com hematoxilina eosina. O G2 apresentou valores de 2,4 e 9,8\% para defeitos maiores e menores com coloração rosa bengala e 0,7 e $9,4 \%$ para defeitos maiores e menores utilizando-se a hematoxilina eosina.

Palavras chave: andrologia, circunferência escrotal, morfologia espermática, Sindi

\section{Spermatic profile Sindhi bulls race}

ABSTRACT. The breeding soundness exam is an important tool in selecting improvers herd bulls, since it allows the identification of problems of infertility and subfertility in males, maximizing the use of these animals in the breeding season and considerably improving the squad pregnancy rates. The purpose of this study was to describe the spermatic profile bulls Sindi, a farm in the town of Johnstown Bridge in the north of Minas Gerais. We evaluated 11 separate two bulls $\mathrm{G} 1<36$ months $(\mathrm{n}=6)$ and G2> 36 months $(n=5)$ raised on pasture. The animals were initially weighed and identified, measured scrotal circumference, where scores a $33.8 \mathrm{~cm}$ to $38.2 \mathrm{~cm}$ and G1 to G2 and subsequently collecting the semen was performed using the eletroejaculator. The ejaculate was subjected to microscopic analysis for evaluating motility swirling and place yielding 78.4 motility values and 65\%, force and turbulence 3.5 and 2.62 .4 for 3 and G1 and $\mathrm{G} 2$ respectively . A sample of this ejaculate was subjected to examination of 
morphology, using the rose bengal dye preparation and wet smear technique using hematoxylin eosin dye. The results obtained for the G1 was $1.85 \%$ of minor flaws and defects greater than $7.75 \%$ in the art with the Rose Bengal dye and $1.15 \%$ and $12.25 \%$ of larger and smaller defects respectively with hematoxylin eosin. G2 had values of 2.4 and 9.8\% for major faults and minor staining with rose bengal and 0.7 and $9.4 \%$ for both major and minor defects using hematoxylin eosin.

Keywords: andrology, scrotal circumference, sperm morphology, Sindhi

\section{Introdução}

A raça Sindi é originária do Paquistão, na região de Kohistan, parte da província Sindh onde é conhecida como Red Sindhi. Os primeiros exemplares da raça chegaram ao Brasil por volta de 1952 e, atualmente, a maior parte do rebanho está presente no nordeste brasileiro. Caracterizados pela pelagem vermelha, variando de tonalidades de acordo com o sexo do animal e o pequeno porte, o Sindi se destaca pela sua rusticidade e tolerância ao calor (Leite et al., 2001). São zebuínos de dupla aptidão, sendo utilizados tanto para produção de carne como de leite, com fêmeas de alta precocidade sexual e boa produção leiteira. Os bezerros nascidos são criados ao pé, totalmente a pasto, e alcançam bom peso a desmama. As matrizes são de alta fertilidade, alcançando taxas de prenhez de até $90 \%$, mesmo com seca antecipada, falta de pasto e temperatura alta, confirmando a rusticidade destes animais (Santos, 2011).

O exame andrológico é uma ferramenta importante na avaliação das funções reprodutivas normais dos machos. $\mathrm{Na}$ seleção dos reprodutores, objetiva-se identificar problemas de subfertilidade ou infertilidade que consequentemente irão comprometer os índices reprodutivos do rebanho tornando assim o exame andrológico imprescindível na detecção de tais alterações (Barbosa et al., 2005). O exame andrológico completo baseia-se na avaliação de todos os fatores envolvidos na função reprodutiva do animal, abrangendo a saúde geral, genital, hereditária, capacidade de copular e de fecundar os oócitos. Tal exame possui indicação na seleção e comercialização de reprodutores, avaliação do potencial reprodutivo, diagnóstico de sub ou infertilidade, diagnóstico de ocorrência da puberdade, preservação do sêmen in vitro dentre outros, segundo o Colégio Brasileiro de Reprodução Animal (CBRA, 1998).

O objetivo deste trabalho foi descrever o perfil espermático de touros da raça Sindi no norte de Minas Gerais, comparando-os com outras raças citadas na literatura.

\section{Material e Métodos}

O estudo foi realizado na fazenda Barra da Vereda, localizada no município de São Joao da Ponte, no norte de Minas Gerais, região de clima semiárido. O rebanho da fazenda é criado a pasto. Avaliou-se 11 animais da raça Sindi, adultos, utilizados para a reprodução na propriedade foram submetidos ao exame andrológico. Os animais utilizados foram separados em dois grupos etários, sendo G1 < 36 meses (n=6) com idade média de 30 meses e $\mathrm{G} 2>36$ meses $(\mathrm{n}=5)$ com idade média de 72 meses. Os indivíduos foram identificados, pesados individualmente em balança mecânica e encaminhados para o tronco de contenção.

A mensuração da circunferência escrotal foi obtida com a fita métrica especifica e todas as informações foram anotadas em fichas de controle.

A coleta de sêmen realizou-se com o uso do eletroejaculador, e imediatamente uma amostra do ejaculado foi submetida à microscopia óptica para o exame físico, observando-se o turbilhonamento espermático (movimento em massa), motilidade espermática (percentual de espermatozóides vivos) e vigor espermática (intensidade de movimento de cauda) utilizandose lâminas previamente aquecidas a 37 graus em mesa aquecedora. Avaliou-se visualmente o aspecto do ejaculado e uma parte da amostra foi diluída em solução de formol salino para a realização do exame morfológico.

A patologia espermática do ejaculado foi realizado em duas técnicas, uma com corante rosa bengala em preparação úmida utilizando-se uma gota de $20 \mu 1$ de amostra e adicionando aproximadamente $10 \mu 1$ do corante rosa bengala a 1,5\% acondicionados entre lâmina e lamínula e secado suavemente com papel toalha a fim de se retirar o excesso do material, e em esfregaço com uso do corante hematoxilina eosina, onde uma 
gota de sêmen é distendida sobre a lâmina para secagem e posteriormente é imersa nos corantes.

A morfologia espermática foi classificada em defeitos maiores e defeitos menores. Para tal exame foram utilizados os critérios de Blom (1973) recomendados pelo Manual para Exame Andrológico do CBRA (1998) em que se contabiliza 200 células por amostra, classificando as anormalidades em defeitos maiores e defeitos menores expressando os valores em porcentagem considerando os padrões morfológicos preconizados por Fonseca et al. (1992).

\section{Resultados e Discussão}

As médias obtidas de peso corporal circunferência escrotal (CE) e características físicas do ejaculado, encontram-se descritas na Tabela 1.

Tabela 1. Variação de peso corporal, CE, exame físico do ejaculado de touros da raça Sindi em 2 grupos etários na região Norte de MG.

\begin{tabular}{lcc}
\hline \multirow{2}{*}{ Variáveis } & \multicolumn{2}{c}{ Médias } \\
\cline { 2 - 3 } & G1 $<36$ meses & G2 $>36$ meses \\
\hline Idade Média, meses & 30 & 72 \\
Peso Médio, kg & 481,7 & 611,4 \\
Circunferência escrotal, cm & 33,8 & 38,2 \\
Características físicas do sêmen: & & \\
$\quad$ Turbilhonamento, 0-5 & 3,0 & 2,4 \\
$\quad$ Motilidade, \% & 78,4 & 65,0 \\
$\quad$ Vigor, 0-5 & 3,5 & 2,6 \\
\hline
\end{tabular}

A circunferência escrotal segundo Gressler et al. (2000) possui alta correlação com a precocidade sexual e idade ao primeiro parto das fêmeas. A mensuração da $\mathrm{CE}$ de touros aos 12 meses de idade é uma importante ferramenta para selecionar os touros que transmitem aos seus produtos, maior fertilidade e ganho de peso.

Segundo Murta et al. (2015) a relação entre peso e CE de touros Sindi mostrou-se variável conforme a faixa etária dos animais, e o perímetro escrotal mensurado de touros Sindi na região norte de $\mathrm{MG}$, foi semelhante ao encontrado em raças taurinas, que no quesito precocidade, se destacam dos zebuínos.

A CE média obtida no estudo foi maior do que a descrita por Folhadella et al. (2006) para zebuínos da raça Gir foi de $28,1 \mathrm{~cm}$ entre de 18 a 24 meses, $32,4 \mathrm{~cm}$ entre animais de 25 a 31 meses e de $32,7 \mathrm{~cm}$ para animais de 32 a 38 meses de idade. Em comparação com a raça Nelore, os valores encontrados por Viu et al. (2006) para animais abaixo dos 36 meses aproximou-se aos descritos neste estudo, atingindo os $34,0 \mathrm{~cm}$ de média nos animais de 25 a 30 meses e 33,9 cm de CE nos animais de 31 a
36 meses. Segundo Pérez Osorio et al. (2012) a CE de touros Guzerá, criados a pasto com idades de 32 a 36 meses, também se demonstraram inferiores aos encontrados neste estudo, obtendose $31,4 \mathrm{~cm}$. Dados descritos por Valentim et al. (2002) mostraram que touros nelores aos 24 meses apresentavam CE de 29,2 cm e touros cruzados Nelore x Europeu, alcançaram médias de CE de 30,9 cm, ainda assim inferiores aos encontrados neste trabalho.

Segundo a tabela de classificação andrológica de touros B. taurus indicus baseada no perímetro escrotal e criada por Fonseca et al. (1997) o G1 composto de animais abaixo dos 36 meses se enquadra na classificação MUITO BOM (32 a 35 $\mathrm{cm})$ para animais de 24 a 36 meses e o G2 se enquadra na classificação EXCELENTE (>38 $\mathrm{cm})$ para animais acima de 60 meses demonstrando a qualidade destes exemplares da raça Sindi.

As médias obtidas das características físicas do ejaculado, foram confrontadas com os padrões seminais desejáveis, sugeridos por Fonseca et al. (1992), no qual a motilidade mínima aceitável é de $50 \%$, vigor 3 e turbilhonamento 3 . No 
presente estudo, obteve-se motilidade média de $78,4 \%$ no G1 e $65,0 \%$ no G2. Estes dados são sugeridos pela literatura e preconizados pelo CBRA (1998). Valores inferiores de motilidade espermática, são relatados por Nogueira et al. (2011) sendo 56,7\% para touros nelore criados no pantanal e $62,2 \%$ para touros nelore do planalto mato-grossense com idades entre 25 e 48 meses em regime a pasto. No mesmo trabalho obteve-se vigor espermático de escore 3,3 para touros do pantanal e 3,4 para touros Nelores do planalto, valores bem próximos do encontrado no ejaculado dos animais deste estudo.

Há que se ressaltar que o método de coleta por eletro ejaculação pode não produzir amostras dentro dos parâmetros fisiológicos da espécie, fato que deve ser ponderado no momento da avaliação segundo o CBRA (1998).

Os dados obtidos do exame de morfologia espermática estão contidos na tabela abaixo (Tabela 2).

Tabela 2. Percentual de defeitos maiores e defeitos menores, utilizando as técnicas de coloração com corante rosa bengala e hematoxilina eosina

\begin{tabular}{lcccc}
\hline & \multicolumn{2}{c}{ Rosa bengala } & \multicolumn{2}{c}{ Hematoxilina eosina } \\
\hline Grupos & Def maior, \% & Drf menor, $\%$ & Def maior, $\%$ & Def menor, $\%$ \\
\hline G1 & 1,85 & 7,75 & 1,15 & 12,25 \\
G2 & 2,4 & 9,8 & 0,7 & 9,4 \\
\hline
\end{tabular}

Nas duas técnicas empregadas para corar as lâminas observou-se que as médias obtidas de células anormais, não ultrapassaram os limites de tolerância estabelecidos pelo CBRA (1998) para qualidade do sêmen que, preconiza um máximo de $10 \%$ de defeitos maiores e $20 \%$ de defeitos menores, não ultrapassando os $30 \%$ de defeitos totais. Entre os defeitos maiores, que são aqueles que afetam a capacidade fecundante do espermatozóide, os principais encontrados foram gotas citoplasmáticas proximais e caudas fortemente dobradas e fortemente enroladas. Já nos defeitos menores, que comprometem a locomoção, porém permitem a fecundação, observou-se maior frequência entre cauda dobrada, cauda enrolada e cabeça decapitada normal.

Galvani et al. (2000) trabalhando com 170 touros Nelore com idade média de 72 meses no estado do Pará, utilizando o corante rosa bengala, obteve médias de 3,93 a $4,05 \%$ de defeitos maiores, superiores as encontradas neste experimento e médias de defeitos menores inferiores, de 3,02 a 4,13\%. Oliveira et al. (2011) realizaram um estudo com touros Brangus, criados extensivamente no Mato Grosso do Sul, e obteve valores de 35,03 e 5,29\% para defeitos menores e maiores respectivamente, em animais de 24 a 48 meses de idade, utilizando a técnica de preparação úmida em contraste de fase. Segundo Freneau (2011), preferivelmente deve-se eleger a técnica de preparação úmida em microscópio de fase ou de contraste diferencial de interferência de fase para exame de morfologia espermática, por apresentarem maior acurácia no exame.

\section{Conclusão}

Os dados obtidos neste estudo mostram que os parâmetros espermáticos de touros da raça Sindi no norte de Minas Gerais estão dentro dos padrões desejáveis para um bom índice reprodutivo no rebanho. Em comparação outras raças o Sindi se mostrou bastante eficiente, com muitos indicativos de sua fertilidade e precocidade sexual.

\section{Referências Bibliográficas}

CBRA. (1998). Manual para exame andrológico e avaliação de sêmen animal. Colégio Brasileiro de Reprodução.

Folhadella, I., Sá, W., Ferreira, A., Camargo, L., Viana, J., Ramos, A. \& Silva, M. (2006). Características andrológicas de touros da raça Gir. Arquivos Brasileiro de Medicina Veterinária e Zootecnia, 58, 809-815.

Fonseca, V., Santos, N. \& Malinski, P. (1997). Classificação andrológica de touros zebus (Bos taurus indicus) com base no perímetro escrotal e características morfo-físicas do sêmen. Revista Brasileira de Reprodução Animal, 21, 36-39. 
Fonseca, V., Vale Filho, V., Mies Filho, A. \& Abreu, J. (1992). Procedimentos para exame andrológico e avaliação de sêmen animal, Belo Horizonte.

Freneau, G. (2011). Aspectos da morfologia espermática em touros. Revista Brasileira de Reprodução Animal, 35, 160-170.

Galvani, F., Costa, E., Torres, C., Bruschi, J., Santos, M. \& Pinheiro, R. (2000). Perímetro escrotal, características físicas do sêmen e morfológicas do espermatozóides de touros Nelore de alta libido comparados com animais de libido infetior. Ars Veterinária, 16, 97-103.

Gressler, S. L., Bergmann, J. A. G., Pereira, C. S., Penna, V. M., Pereira, J. C. C. \& Gressler, M. G. d. M. (2000). Estudo das associações genéticas entre perímetro escrotal e características reprodutivas de fêmeas Nelore. Revista Brasileira de Zootecnia, 29, 427-437.

Leite, P. M., Santiago, A., Navarro Filho, H., Albuquerque, R. \& Leite, R. (2001). Sindi: Gado vermelho para o semi-árido. EMEPAPB; Banco do Nordeste.

Oliveira, L. Z., Oliveira, C. S., Monteiro, F. M., Martins Hossepian de Lima, V. F., Lima, F. M. \& Costa, M. Z. (2011). Efeito da idade sobre as principais características andrológicas de touros Brangus-Ibagé criados extensivamente no estado do Mato Grosso do Sul-Brasil. Acta Scientiae Veterinariae, 39, 18.

Pérez Osorio, J., Marc, H., García Bergmann, J. A., Santana, A. S. \& Souza, F. A. (2012). Desenvolvimento testicular e puberdade em machos da raça guzerá da desmama aos 36 meses de idade criados no cerrado mineiro. Revista de Medicina Veterinária, 9-24.

Valentim, R., Arruda, R. P., Barnabe, R. C. \& Alencar, M. M. (2002). Biometria testicular de touros Nelore (Bos taurus indicus) e touros cruzados Nelore-europeu (Bos taurus indicus $\mathrm{x}$ Bos taurus taurus) aos 20 e 24 meses de idade. Brazilian Journal of Veterinary Research Animal Science, 39, 113-120.

Viu, M., Magnabosco, C., Ferraz, H., Gambarini, M., Oliveira Filho, B., Lopes, D. \& Viu, A. (2006). Desenvolvimento ponderal, biometria testicular e qualidade seminal de touros Nelore (Bos taurus indicus) criados extensivamente na região centro-oeste do Brasil. Archives of Veterinary Science, 11, 5357.

Recebido em Novembro 11, 2015

Aceito em Dezembro 15, 2015

License information: This is an open-access article distributed under the terms of the Creative Commons Attribution License, which permits unrestricted use, distribution, and reproduction in any medium, provided the original work is properly cited. 